\title{
Théologiques
}

\section{À Pierre Ouellet, un théologien reconnaissant}

\section{Olivier Bauer}

Volume 20, numéro 1-2, 2012

URI : https://id.erudit.org/iderudit/1018868ar

DOI : https://doi.org/10.7202/1018868ar

Aller au sommaire du numéro

Éditeur(s)

Faculté de théologie et de sciences des religions, Université de Montréal

ISSN

1188-7109 (imprimé)

1492-1413 (numérique)

Découvrir la revue

Citer ce compte rendu

Bauer, O. (2012). Compte rendu de [À Pierre Ouellet, un théologien reconnaissant]. Théologiques, 20(1-2), 479-488.

https://doi.org/10.7202/1018868ar d'utilisation que vous pouvez consulter en ligne.

https://apropos.erudit.org/fr/usagers/politique-dutilisation/ 
NOTE DE LECTURE

\title{
À Pierre Ouellet, un théologien reconnaissant ${ }^{1}$
}

\author{
Olivier BAUER* \\ Théologie \\ Université de Montréal (Canada)
}

Pierre Ouellet (philosophe: 1950-) est un passeur (au double sens de celui qui permet de passer vers un autre endroit et de celui qui adresse une passe dont on se doit de profiter). Pierre Ouellet est un penseur qui compte, au Québec, au Canada et même dans le monde. En témoignent ses fonctions universitaires: il est titulaire de la chaire du Canada en esthétique et poétique, professeur titulaire au département d'études littéraires de l'Université du Québec à Montréal, membre régulier du Centre interuniversitaire d'études sur les lettres, les arts et les traditions, professeur invité au Mexique, au Brésil, en France et au Japon. En témoigne sa bibliographie: il a publié une quarantaine d'ouvrages tant de poésie (premier recueil en 1989: Sommes) que d'essais (premier ouvrage en 1990: Chutes. La littérature et ses fins). En témoignent les nombreux prix et distinctions qui reconnaissent sa pensée: il est lauréat de deux prix du Gouverneur général, il a été élu à l'Académie des lettres du Québec, il est membre de la Société Royale du Canada, etc. ${ }^{2}$

Dans son ouvrage Sacrifiction, il offre sa pensée sur le sacré et la fiction, sur le sacré comme fiction, sur la fiction comme sacré, sur «Dieu ou

* Olivier Bauer est professeur agrégé en théologie pratique à l’Université de Montréal. Ses recherches actuelles portent sur le rôle des six sens dans le développement de la foi et l'alimentation dans le christianisme. Il a récemment publié (2011) Une théologie du Canadien de Montréal, Montréal, Bayard.

1. Note de lecture de P. Ouellet (2011) Sacrifiction: sacralisation et profanation dans l'art et la littérature, Montréal, VLB. Les chiffres entre parenthèses dans le texte renvoient à la pagination du livre.

2. Informations trouvées sur le site Internet de la chaire du Canada en esthétique et poétique: <http://www.esthetiqueetpoetique.uqam.ca/titulaire.htm>.

(C) Revue Théologiques 2012. Tout droit réservé. 
quoique ce soit du genre» (119). Matériellement, l'ouvrage est un livre de 392 pages dans une belle édition de VLB éditeur. Il se présente comme le $28^{\mathrm{e}}$ titre de la collection "Le soi et l'autre» que Pierre Ouellet dirige personnellement.

Sur la couverture du livre figure la reproduction d'un montage de Christine Palmiéri, intitulé Livre-autel et daté de 2011³. L'image donne sans aucun doute le ton et le sens de l'ouvrage. Je la regarde et j'y vois un vieux livre épais, relié en peau, en bon état, fermé (a-t-il été seulement ouvert?); sur le livre repose une tête coupée, celle d'une statue en bois polychrome, celle d'un homme jeune, beau, la bouche fermée, les yeux, noirs, un peu bridés, grand ouverts, le regard fixe; avec ses cheveux ondulés et ses pommettes saillantes, il pourrait être un Christ ou un ange; de son cou, de la commissure de ses lèvres et de son nez coulent deux fois trois filets de sang rouge; ils dégoulinent sur la tranche et sur le dos du livre; ils se rejoignent pour former une tache visqueuse, poisseuse, irrégulièrement arrondie. Tout est montré, tout est dit: ensemble, l'image et l'écrit; ensemble, l'art et le sacrifice; ensemble, la fiction et le sacré; et cette référence au christianisme.

Dans le livre, Ouellet déploie une pensée originale et féconde sur la «sacralisation et [la] profanation dans l'art et la littérature » (sous-titre de l'ouvrage) essentiellement contemporains, une pensée qu'il organise en deux parties d'égales longueurs, la première intitulée: "La part de Dieu» (avec une majuscule) et sous-titrée "Fictions du sacré », et la seconde: «Tonalités du verbe» (avec une minuscule) et sous-titrée «Tonus et virtus». Ouellet y écrit (c'est à dire qu'il montre ce qu'il dit) comment s'articulent le sacré (du côté de Dieu ?) et la fiction (du côté du verbe ? à moins que ce ne soit l'inverse), comment des artistes voient et entendent le monde, comment des plasticiens et des poètes disent et montrent le monde, comment « la perte de toute vision d'ensemble et de toute voix commune laisse place à l'émergence de "vocalités" et de "visualités" inédites où peuvent apparaitre des modes inattendus de socialité et de sacralité » ( $4^{\mathrm{e}}$ de couverture). Ouellet ne se contente pas de parler des artistes. Il leur donne la parole, il leur donne l'image, plaçant dix-sept illustrations (malheureusement en noir et blanc) d'œuvres et d'installations (elles sont toutes dans la première partie, comme si le verbe ne pouvait être exprimé qu'en mots) et truffant son texte d'extraits de poésie et de fiction. Les œuvres d'art servent

3. Une image de la couverture figure sur le site Internet de l'éditeur: <http://www.edvlb. com/sacrifiction/pierre-ouellet/livre/9782896494026>. 
tantôt de point de départ à la pensée de Ouellet; elles se situent tantôt à son point d'aboutissement ${ }^{4}$.

Voici comment moi, Olivier Bauer (théologien: 1964-), je l'ai pris, compris, certainement parfois mépris, enfin repris, à moi, avec moi, pour moi. Le voici écrit dans un ton, sur un rythme que j'ai voulu laisser proche ou, plutôt, cherché à rendre proche de l'écriture de Ouellet. Pour mieux rendre compte de la manière dont il énonce sa pensée, finalement pour mieux lui rendre justice. À la poésie du philosophe, il m'a semblé que devait correspondre (et répondre) celle du théologien.

\section{Ce qu'il faut!}

Il faut une révélation pour être sauvé de l'oubli, une rédemption pour être sauvé du fini.

\section{Pourquoi le faut-il ?}

D’abord parce que même si Dieu semble mort, même si «ses nombreuses incarnations et manifestations, traces, spectres, fantômes, revenants » (23) sont encore, toujours, de nouveau, à nouveau mis à mort, il y a pourtant encore, toujours «l'écho de son existence» (179), «ce grand Dehors » qui est en même temps «cet obscur Dedans» (17), un «Rien qui prend les formes du Divin» (20), une "question du Sacré» (21).

Ensuite, parce que "les choses ne sont pas: elles se produisent, elles arrivent $[\ldots]$ De plus loin qu'où elles sont, et plus loin qu'où elles vont » (36); au-delà de ce qui est, mais déjà entre ou parmi ce qui est, il y a ce qui pourrait être et ce qui aurait pu être, «l'éventuel et l'alternatif, le conditionnel et le contre-factuel» (117).

Encore parce que «paroles et images doublent le réel» (36), qu'elles le doublent même doublement: en le dépassant (elles vont plus loin encore) et en lui conférant une épaisseur (un sens qu'à la fois elles dispensent et dont elles dispensent, d'où parfois leur non-sens).

Enfin, parce que, même si "on ne croit plus, sinon en creux ou par défaut» (203), il y a un fondamental «besoin de confiance », un croire,

4. Sans que ni Ouellet ni son éditeur ne le signale explicitement, j'imagine que Pierre Ouellet s'est nourri du colloque Sacrifictions. Profanation et sacralisation en art et en littérature, qu'il avait organisé en collaboration avec le Musée d'art contemporain de Montréal entre le 12 et le 17 avril 2010, auquel j'ai assisté. 
mais sans credo; une foi, mais «en soi, sans objet précis, vécue de manière intransitive» $(179)$.

\section{Comment le peut-on?}

Grâce aux artistes et aux poètes (quelques plasticiens: Eruoma Awashish, Jean Fabre, David Moore, Christine Palmiéri; de nombreux poètes dont Augustin, Bibitte Bacon, Philippe Beck, Jean-Marc Desgent, Antoine Émaz, Alain Fleischer, Isabelle Garron, Gérard Manley Hopkins, Max Loreau, Stéphane Mallarmé, Jean-Paul Michel, Marcel Moreau, Brice Petit, Serge Pey, Guez Ricord, Arthur Rimbaud, Richard Robertson, Angelus Silesius, Salah Stétié, Guy Viarre, Antoine Volodine, Patrick Watteau), qui rejouent le moment originel, fondateur, où le corps régule en son sein l'extérieur et l'intérieur, le monde qui le pénètre par les yeux (impression) et le souffle vital qu'il expire par la bouche (expression). Grâce aux artistes que meuvent et émeuvent deux passions esthétiques: "l'enthousiasme et le dépit », «l'eu-phorie et la dys-phorie », l'enlèvement (la sacralisation) et le soulèvement (la profanation) (182-183). Grâce aux artistes qui donnent à voir ce qu'ils entendent, à entendre ce qu'ils voient, qui procèdent inlassablement à l'autopsie minutieuse du « Grand Cadavre en décomposition du Dieu des religions» (21).

Grâce à la vue et à l'ouïe qui font entrer l'être humain dans le sens de l'autre (alteroperception), quand la dévoration et la copulation laissent l'être humain dans le seul sens de soi (proprioperception) (56). Grâce à la vue et à l'ouïe qui introduisent une distance de soi à l'autre et de soi au tout autre, là où la dévoration et la copulation produisent la fusion du soi et de l'autre, du soi et du tout autre (57-58). Grâce à la vue et à l'ouie qui provoquent fissures, ruptures, brisures entre le profane et le sacré, entre la nature et la culture, entre l'individu et la société, entre l'âme et le corps. Grâce à la vue et à l'ouie qui suscitent, par-delà la distance, le désir d'une couture, d'une suture, d'une reliure à l'autre (régime social) comme au tout autre (régime religieux) (57).

" "Voir" pousse à "faire voir". "Entendre", à "faire entendre" " (57). Mais voir et faire voir quoi? Entendre et faire entendre quoi? Car il y a langage et langage. Il y a "l'usage dogmatique, idéologique, juridique du langage: l'Église, l'État, le Droit» (59) qui nie toute distance et tente de colmater toute brèche par la contrainte et le lien. Et il y a «l'usage poḯtique et esthétique de l'image et de la parole: l'Art, la Littérature, la 
Poésie» (59) qui tient à distance et ravive «la scission ou la fissure originelle». Et concrètement:

- Il y a le langage du mythe. En amont de toute rupture. Qui fait remonter à l'origine, au moment précis de «l'hominisation». Qui délie, lie et relie. À la fois déboîtement et emboitement, rupture et ligature, arrachement et attachement (60).

- Il y a le chamane. Qui conjoint le croire et le savoir, la «foi aveugle» et la "clairvoyance de l'esprit », la "confiance infinie » et l' "hyperlucidité de la conscience", bref «ce qu'on a voulu quant à nous séparer radicalement en science et religion» (106). Qui surmonte à l'intérieur même de son corps les «institutions du Savoir, de la Croyance et du Pouvoir» (108-109).

- Il y a la bouche au double usage: par quoi le monde entre en nous (inspiration, incorporation) et grâce à quoi nous rendons le monde à luimême (amplification, prononciation) (112).

- Il y a les bâtons à message, les tshissinuashitakana qui permettent aux Innus d'informer d'autres Innus; discrets messages visuels, ils demandent «un œil aiguisé pour les apercevoir» (144); comme eux, il y a «les mots du poème et les images de l'art» qui nous permettent de «suivre la route des esprits, ou la piste des ancêtres sans nous égarer» (145).

Enfin, surtout, il y a Dieu: non pas celui que l'on imagine, mais le «grand Imageant ou Imaginant» (216), «l'imageance sans borne» (217); plus qu'une image, un "imageable », "une vue à vide» (231); celui qui est "au-delà de tout attribut particulier» (167). Dieu, diapason qui donne «son Ton, sa Tonalité, sa Tonicité... son Air » (165) au "chef des souffles » (le chamane). Dieu, métronome qui donne la mesure au "maître des rythmes» (le poète). Dieu qui leur donne de faire ainsi «les seules communautés possibles» (166), celles qui s'accordent.

\section{Et pour quoi le faut-il?}

"Les icônes ont été souillées », "les idoles sont brisées ", «l'imaginaire est fiévreux» (203). Alors, il faut l'image poétique «à distance égale de toute idolâtrie et toute iconoclastie, de toute fétichisation du visible et tout interdit de la représentation» (210). Alors, il faut l'image poétique, "pure motilité » qui jamais ne s'arrête, feu et eau, «flamme qui monte ou pluie qui tombe» (205), voix qui brûle et parole qui rafraîchit. Alors il faut le poème qui certes "ramasse les morceaux" des idoles cassées, mais pour mieux «les disperser, les disséminer, les jeter aux quatre vents» (207). 
Alors il faut le poème qui certes est religio, mais religio sauvage, indomptable où Dieu est un loup, non pas un chien, où la langue est un forban, un élan, un "emportement vers le grand "dehors" " (295). Alors, il faut le poème qui délie, qui libère, qui corrige aussi, ni par le plus ni par le trop, seulement par le moins, par le blanc, par le peu, par le rien.

"Toute expérience littéraire est Gnôsis, Noésis, Poïesis; connaître, penser, créer» (303). Gnôsis au sens de l'acte même de connaître, de conaître, d'une rencontre intime, d'une "double naissance des choses à soi et de soi aux choses ", d'une "double venue du langage à l'être et de l'être au langage» (305).

Il faut prendre la parole pour prendre de la distance. Il faut poser un regard pour poser un diagnostic. Il faut voir et nommer. Encore l'image et la parole. Il ne s'agit pas, plus, de savoir ce qui est vrai en soi (c'est là le but de l'épistémè), mais de connaître ce qui est bon pour soi (c'est ici le but de la gnôsis). Et qu'est-ce qui est bon pour soi ? L'enthousiasme, dans son sens premier de «transport divin» (339), qui nous «ex-hausse» et nous «ex-alte» (339). Mais encore? Qu'il y ait un Dieu, même s'il nous tourne le dos, "occupé qu'il est à regarder du côté opposé à celui de l'homme » (348), même si son verso est la seule face qu'il nous montre. On doit lui en vouloir, légitimité de l' «insoutenable irritation» (348). Mais on peut aussi en profiter pour l'accrocher. Car il peut nous porter sur sa croupe et nous emporter "plus loin qu'où nous ne pourrions jamais aller par nous-même » (340), par-delà la vie et la mort. Car il peut nous rendre à notre Être, un Être «transhumain», "ultramondain» et même «outre-divin» (341). Dieu «ne se parle qu'en langue», "sa chair étant verbe» (341).

Mais le Verbe a été sacrifié et la langue, dans une "transcendance immonde» (345), profane ce qu'elle sacralise, déteste les images qu'elle adore, voue aux gémonies le langage auquel elle voue un culte. Mais le Verbe a été ressuscité et la langue, comme un levier, soulève "dans les airs", "jusqu'au ciel » le "grand cadavre de Dieu». Mais le Verbe est retombé «sur la tête des apôtres le jour de la Pentecôte» (349) et la langue, comme une catapulte, le fait éclater pour qu'il « retombe sur nous en langues de feu» (349), mettant «la langue de Dieu dans la bouche des hommes» (348).

\section{Ce que j'en garde, ce que j'en transmets!}

Théologien protestant qui m'intéresse à la manière dont le croire vient aux êtres humains, j'ai beaucoup reçu de ma lecture de cet ouvrage. Une lecture 
lente, mais passionnante et passionnée, au double sens du désir (passion amoureuse) et de la souffrance (Passion du chemin de croix). Lecture lente, car l'écriture de Ouellet est dense, rigoureuse et riche de jeux de mots (en particulier quand il travaille l'étymologie des mots pour en ouvrir le sens); lente, car la culture de Ouellet est grande (j'avoue que je ne connaissais pas la plupart des artistes qu'il évoque) et qu'il présuppose que son lecteur la partage. Mais lecture rendue passionnante et passionnée par et pour les liens tissés avec ce que je sais de Dieu, des êtres humains du monde et des relations qu'ils entretiennent ou n'entretiennent pas; par et pour les liens avec ce que je crois de Dieu, des êtres humains, du monde et de moi et des relations que nous entretenons et n'entretenons pas.

Pierre Ouellet m'a notamment dit ou redit:

- Le besoin d'une réflexion fondamentale sur D/dieu (Ouellet l'écrit souvent avec un «D", parfois avec un « $\mathrm{d}$ »; rarement, me semble-t-il, avec un « $\mathrm{X}$ ») et sur les noms qu'on peut lui attribuer ou plutôt dont on peut l'affubler.

- L'espace de l'interprétation entre un monde rassurant qui nous adresse des données sensibles et nous qui devons en tirer du sens: "Nous ne sommes pas seuls, nous ne sommes pas abandonnés, nous ne sommes pas seuls, le monde s'adresse à nous, le monde nous apparaît, le monde nous "parle”, si nous savons ouvrir l'œil, tendre l'oreille " (111).

- Le rôle essentiel de la vue et de la parole (deux sens que toujours Ouellet apparie quand bien même ils fonctionnent dans des directions opposées) dans le processus d'hominisation. Ils introduisent de la distance entre soi et l'autre, entre soi et l'Autre, permettant ainsi une relation de réciprocité: "Je ne m'associe à l'autre que par et dans les vues et les voix que l'on s'échange: la vue que l'on se donne l'un de l'autre, la voix que l'on se donne l'un à l'autre» (57).

- La capacité des sens, de la vue et de l'ouie, de l'image et de la parole, à percevoir le visible comme l'invisible, l'audible comme l'inaudible, à transmettre le montrable comme l'immontrable, le dicible comme l'indicible.

- La force des images et des poèmes qui montrent et disent Dieu, l'être humain, le monde, la nature, ce qu'ils font les uns des autres et les relations qu'ils entretiennent ou n'entretiennent pas. Ces images sont-elles outrancières? Sont-elles outrageantes? Souvent, sans aucun doute. Mais qu'est-ce que l'outrage, qu'est-ce que l'outrance pour celui qui croit que Dieu a été crucifié ? De quoi faut-il avoir peur: de la profanation ou de la sacralisation? 
- La fécondité des métaphores bibliques: «le poète est une sorte d'Abraham penché sur la nuque du jeune Isaac qu'il s'apprête à raccourcir... sur les ordres de quelque Dieu assisté par quelque Diable» (274); "l'écriture est transfiguration du Fils en Père et inversement par la Parole qui les sépare et les unit en même temps » (278); "le vent paraclet» (280), «l'économie de la Grâce» (281), etc.

Et pour à mon tour introduire la distance du dialogue, j'aimerais modestement adresser quatre remarques à l'ouvrage de Pierre Ouellet.

1. J'ai failli refermer le livre à la seconde page, après avoir lu que l'imagination est "la capacité de produire des images en l'absence de stimuli sensoriels précis, identifiables, reconnaissables, sans le secours des choses physiques dont elles sont censées être le reflet» (11), que «l'homme va plus loin [que «le lion, le tigre, le loup»]: il imagine ce dont aucun bruit des feuilles n'est l'indice, ce qu'aucune odeur n'annonce, ce dont rien n'est la prémisse, ce que rien n'anticipe» (11-12). Or il me semble que toutes nos images, et même celles de Dieu, dépendent de nos perceptions sensorielles. L'imagination serait donc plutôt la capacité à relier, à réarranger, à transformer, à sublimer les stimuli que nous percevons et que nous élaborons ( «la carte n'est pas le territoire» et nous ne percevons pas «la réalité ", nous la reconstruisons au travers des perceptions conscientes et inconscientes forcément déformées que nous en avons). Alors, même la Parole de Dieu n'est plus la Parole de Dieu, mais une parole sur Dieu, une parole que nous prêtons à Dieu; alors, même nos images de Dieu ne sont jamais que des images partielles, subjectives d'un Dieu qui reste toujours à la fois caché, ou "de dos", et révélé comme "Dieu de peu» en Jésus Christ.

2. Par conséquent, je refuserais moi aussi «le théologique» si, comme Ouellet le définit, il était la «logique de dieu» (345). Mais la théologie ne peut jamais prétendre démontre la logique de Dieu; elle peut tout au plus mettre en évidence une certaine logique dans ce que les êtres humains croient (ou disent qu'ils croient ou montrent qu'ils croient) à propos de leur Dieu. Théologien, c'est ce que je m'efforce de dire, d'écrire, de montrer, de faire.

3. Ma lecture me laisse avec l'impression que pour Ouellet, les restes de Dieu ou les empreintes du Sacré seraient en quelque sorte déjà-là, toujours-là, dans ces «données sensibles que nous adresse le monde» (55), celles que les artistes et les poètes (mais les philosophes aussi) déchiffrent pour nous. Mais alors quelle est la place de l'athéisme ou de l'incroyance? Seraient-elles seulement au mieux des erreurs de bonne foi, au pire des 
formes de mauvaise foi ? Un aveuglement, une surdité, le refus d'admettre ce qui serait pourtant évident? Il est vrai que Ouellet se situe du côté de la gnôsis, et qu'il ne prétend pas dire ce qui est vrai en soi. Il n'empêche. Quand il affirme par exemple que «le poids d'un Dieu mort est plus lourd sur nos épaules que celui d'un Dieu vivant» (364), il devrait l'écrire "pour soi », pas "pour nous". Il devrait écrire: «Le poids d'un Dieu mort est plus lourd sur mes épaules que celui d'un Dieu vivant.»

4. Je comprends que Ouellet pense le sens du toucher dans l'intimité $\mathrm{du}$ «baiser que le poète donne à Dieu sur les lèvres de la femme aimée » (encore que je regrette que la sensualité reste seulement métaphorique). Je comprends moins qu'il poursuive en liant toucher et sacrifice: «Le baiser que le poète donne à Dieu sur les lèvres de la femme aimée confère à cette dernière le caractère sacré de "bouche émissaire" » (232), émissaire étant entendu au triple sens de "messager", de "voie d'évacuation" et de "celui ou celle sur qui l'on fait retomber les torts de tous, le chargeant de tous les péchés, et dont la mise à mort permet seule l'expiation» (232). Que Ouellet valorise la dimension du sacrifice, comme don parfait, relève de son choix, de son droit. Qu'il la retrouve dans les artistes qu'il convoque, je n'en doute pas. Mais, signe qu'il s'agit peut-être bien là d'un choix, Ouellet éprouve pour une fois, le besoin de justifier. Dans le «culte sacrificiel, plongeant ses racines dans le sacré archaïque le plus puissant» (233), «Roger Bastide verrait là une résurgence ou une survivance de ce qu'il appelle le "sacré sauvage". [...] Gérard Bucher, quant à lui, y verrait sans doute le retour inattendu au fétichisme primitif. [...] René Girard y décèlerait une régression vers la violence la plus crue, non encore régie par le mécanisme du bouc émissaire messianique.» (233). Mais ce que l'anthropologue, le philosophe et l'historien voient comme «l'indice d'un sentiment préreligieux du monde, ancré dans le sacré idolâtre », Ouellet le voit comme un «sacré postreligieux ou posthistorique» dont la visée est «de dénouer, de détacher, de désarmer l'homme et la femme de leur propre histoire et de leur monde, dont on annonce la fin. » (233). De manière peut-être inattendue, le théologien chrétien que je suis penche plutôt du côté de l'anthropologue, du philosophe et de l'historien. Pourquoi faudrait-il que la libération passe par un sacrifice? Pourquoi ce besoin d'un bouc émissaire? Souvent Ouellet évoque (ou invoque?) en sa faveur ce qu'il juge être deux sacrifices bibliques: la ligature d'Isaac et la crucifixion de Jésus. Possibles traces d'une culture catholico-québécoise où l'on sacrifie le Christ, eucharistie après eucharistie, où l'on offre, hostie après hostie, son corps à la dévoration. Lecture commode mais partiale de ces deux 
récits bibliques. Car Abraham n'a pas égorgé Isaac et Jésus a fini exécuté par des hommes et ressuscité par Dieu. Pour ma part, je crois de moins en moins à l'interprétation sacrificielle de la mort de Jésus. Rachat? Rédemption? Mais qui paye quoi à qui ? Serait-ce Dieu qui s'offrirait son Fils pour calmer sa colère? L'offrirait-il au Mal, au Diable, au Satan pour solde de tout compte? Ferait-il payer à Jésus le prix de ses ambitions? À un tel Dieu, je ne voudrais pas croire. Et s'il faut profaner, c'est peut-être d'abord cette image-là de Dieu; c'est peut-être d'abord la facilité avec laquelle nous faisons du sacrifice une nécessité. 